\title{
A Thermal Study of Tumor-Treating Fields for Glioblastoma Therapy
}

\author{
Nichal Gentilal, Ricardo Salvador, and Pedro Cavaleiro Miranda
}

\section{Introduction}

\subsection{Electromagnetic Radiation and Matter}

The interaction between electromagnetic (EM) radiation and biological tissues has been a subject of study for a long time. It is known that the effects of these interactions depend on factors such as intensity, frequency, and duration of the field. Due to the wide range of possible outcomes, EM radiation has been used in very different areas.

In medicine, techniques that work at a low frequency range $(<10 \mathrm{kHz})$ such as transcranial magnetic stimulation (TMS), transcranial alternating current stimulation (tACS) and transcranial/transcutaneous spinal direct current stimulation (tDCS/ tsDCS) are employed in diagnosis and treatment of some neurological and psychiatric conditions. As the frequency increases, the main effect of these fields at the cellular level is no longer membrane depolarization, and thus stimulation does not occur.

As the wavelength gets shorter, the medical applications start to change. For instance, frequencies of a few $\mathrm{MHz}$ and higher are typically used to produce anatomical images of the human body in techniques such as magnetic resonance imaging (MRI) and microwave imaging (MWI). In other medical procedures, such as hyperthermia and ablation techniques, the main goal is not to diagnose but rather to kill tumoral cells by overheating them.

\footnotetext{
N. Gentilal $(\bowtie)$. P. C. Miranda

Instituto de Biofísica e Engenharia Biomédica, Faculdade de Ciências da Universidade de Lisboa, Campo Grande, Lisboa, Portugal

e-mail: ngentilal@fc.ul.pt

R. Salvador

Neuroelectrics, Avinguda Tibidabo, 47 bis, Barcelona, Spain
} 
For frequencies higher than that of the visible light, EM radiation is strong enough to ionize cells and affect chemical bonds. This type of radiation finds its most valuable use in cancer treatment where techniques such as radiotherapy have been developed based on this type of interaction. Another common application of such type of radiation is in diagnosis (e.g., X-ray imaging, mammography, computed tomography (CT), etc.).

There is a frequency range, in the hundreds $\mathrm{kHz}$ region, that is too high to stimulate tissues but too low to cause significant temperature increases. Up until the beginning of the century, it was commonly believed that fields with this frequency did not have any biological effect on tissues, and thus, they were practically neglected for medical purposes. However, in 2004 the in vitro studies of Kirson et al. [1] revealed for the first time a potential application for this type of radiation: disruption of cancer cell replication. These findings led to a new cancer treatment technique named tumor-treating fields (TTFields).

\subsection{Tumor-Treating Fields}

TTFields consist in applying an alternating electric field (EF) with a frequency between 100 and $500 \mathrm{kHz}$. The mechanisms of action of this technique are still not fully understood. The first hypotheses suggested a two-stage action: during metaphase, these fields could disrupt mitotic spindle formation by acting on cells' highly polar structures, and during cytokinesis, they could affect correct cell division by leading to membrane blebbing $[1,2]$. However, more recent studies presented some calculations that indicate that these hypotheses might not fully explain how TTFields affect the mitotic process [3], and new possible mechanisms have been suggested since [4,5]. An example of the latter are the calculations done by Berkelmann et al. [5] that propose that the high energy deposited at the cleavage furrow might kill tumoral cells by overheating them during telophase and cytokinesis. Despite these uncertainties, the minimum EF intensity that should be induced at the tumor bed to achieve an antimitotic effect is well-established to be $1 \mathrm{~V} / \mathrm{cm}$. Furthermore, application of these fields in two perpendicular directions alternately also proved to increase the number of cells targeted [1].

TTFields are FDA-approved for the treatment of recurrent and newly diagnosed cases of glioblastoma multiforme (GBM), following the outcomes of the EF-11 [6] and EF-14 [7, 8] clinical trials, respectively. More recently, TTFields were also approved for the treatment of malignant pleural mesothelioma after the results from the STELLAR clinical trial [9] showed a treatment improvement when this technique was applied jointly with chemotherapy.

Post hoc analysis of the results from these trials allowed to identify some of the most significant factors that could affect treatment outcome. A detailed analysis on the relation between TTFields inhibitory effect and the intensity of the electric field at the tumor site was performed by Ballo et al. [10] using data from the EF-14 trial. For this study, the median overall survival (OS) and the progression-free survival 
(PFS) were significantly higher for the patients in which the EF was greater at the tumor bed. This corroborates what was already seen in in vitro cell cultures in the study of Kirson et al. [1]. On the other hand, other studies investigated the correlation between treatment time and OS and PFS. Kanner et al. [11] showed that in patients with recurrent GBM, a daily compliance of at least $75 \%$ leads to significantly better treatment outcomes. In newly diagnosed GBM cases, where TTFields are applied in combination with temozolomide, this value drops down to $50 \%$ as discussed in [12]. The idea of an extended treatment time is further supported by the study of Giladi et al. [13] in which it was shown that slowly dividing cells are more likely to be affected by the electric fields the longer they are applied. Despite this clear need of maximizing patient's exposure time to TTFields, it is also important to address how this technique could affect biological tissues due to the temperature increases as a result of the Joule effect. The purpose of this work is to quantify these rises and predict the thermal impact using a realistic head model. Additionally, we suggest new possible ways to apply the fields based on the results we obtained.

\subsection{The Optune Device}

Optune (https://www.optune.com) is the device developed by Novocure (https:// www.novocure.com) to treat GBM with TTFields. It is composed of an EF generator connected to four transducer arrays that work in pairs. The arrays are strategically placed on patient's shaved scalp to increase the electric field at the tumor using the NovoTAL System. This FDA-approved software uses MR images to create personalized treatment maps by adapting the configuration of the paired arrays. The importance of individualized planning was clearly shown in the work of Wenger et al. [14]. In this computational study, the maximum electric field at the tumor almost doubled when the arrays were specifically adapted for each patient. These conclusions were further corroborated by Korshoej et al. [15] in which the authors evaluated the best array positioning for different tumor locations.

Each array consists of a matrix of $3 \times 3$ interconnected transducers with temperature sensors. Each transducer is a ceramic disk with a diameter of $18 \mathrm{~mm}$ and a thickness of $1 \mathrm{~mm}$. The Optune device works at $200 \mathrm{kHz}$ and injects around $900 \mathrm{~mA}$ of current amplitude in two perpendicular directions alternately, with a switching time of $1 \mathrm{~s}$. To allow current to flow from the capacitive transducers to the scalp, a thin layer of conductive hydrogel is used between them.

As noted in several works [2, 6-8] and summarized by Lacoutre et al. [16], the occurrence of dermatologic effects is the main side effect reported so far during TTFields therapy. These might be explained by chemical irritation in the skin due to the use of the hydrogel, moisture, mechanical trauma from shaving, or array removal, for example. To avoid burns, Optune controls the injected current to keep transducers' temperature below $41^{\circ} \mathrm{C}$. 


\section{Methods}

\subsection{The Realistic Human Head Model}

A realistic human head model was used to perform the studies here presented. Creation of this model to study the electric field in the cortex during tCS is described in detail in [17]. Briefly, MR images of the Colin27 template with a resolution of $1 \times 1 \times 1 \mathrm{~mm}^{3}$ were retrieved from BrainWeb. Segmentation was performed using BrainSuite, and as a result five different surface meshes were created delimiting different tissues: scalp, skull, cerebrospinal fluid (CSF), gray matter (GM), and white matter (WM). Mimics was then used for some corrections and model improvement. The latter included representation of the superior orbital fissures and optical foramina and addition of the lateral ventricles that were considered to be filled with CSF.

The adaptation of this model for TTFields studies is described in [18]. Shortly, during model manipulation in Mimics, a virtual lesion was added in the right hemisphere of the brain, near the lateral ventricle, more or less at the same distance to the anterior and posterior regions of the head. This lesion intended to represent a GBM tumor, and it consisted in two concentric spheres: one that represented a necrotic core, with a diameter of $1.4 \mathrm{~cm}$, and the other that represented an active shell, with a diameter of $2 \mathrm{~cm}$. At this stage, the four transducer arrays were also added to the model. One pair was placed over the anterior and posterior regions of the head (AP configuration) and the other pair over the left and right temporal and parietal regions (LR configuration). Although this array positioning is not optimized for this virtual lesion, it represents a possible layout for a real patient. To fill in the gap between the transducers and the scalp, a layer of gel with variable thickness ( $0.7 \mathrm{~mm}$ median) was added to the model. After these modifications were made, a volume mesh was generated (Fig. 1), which consisted of around $2.3 \times 10^{6}$ tetrahedral elements with an average element quality of 0.50 . This mesh was then imported to COMSOL Multiphysics to run the simulations.

\subsection{Heat Transfer in TTFields: Relevant Mechanisms}

Under normal physiological conditions, the human body core temperature is kept between 36 and $37.5^{\circ} \mathrm{C}$ over a wide range of environmental circumstances [19]. In contrast, skin temperature can vary significantly as a function of the surroundings. In fact, heat flows from the most internal organs and tissues to the skin, where it is dissipated to the air [20].

The interplay between heat production and heat loss is a complex system that allows the body to regulate itself in order to maintain its activity. In this section, an overview of the most significant heat transfer mechanisms for a patient undergoing TTFields therapy is discussed. In this context, the main cooling mechanisms include conduction, convection, sweat, radiation, and blood perfusion. On the other hand, 


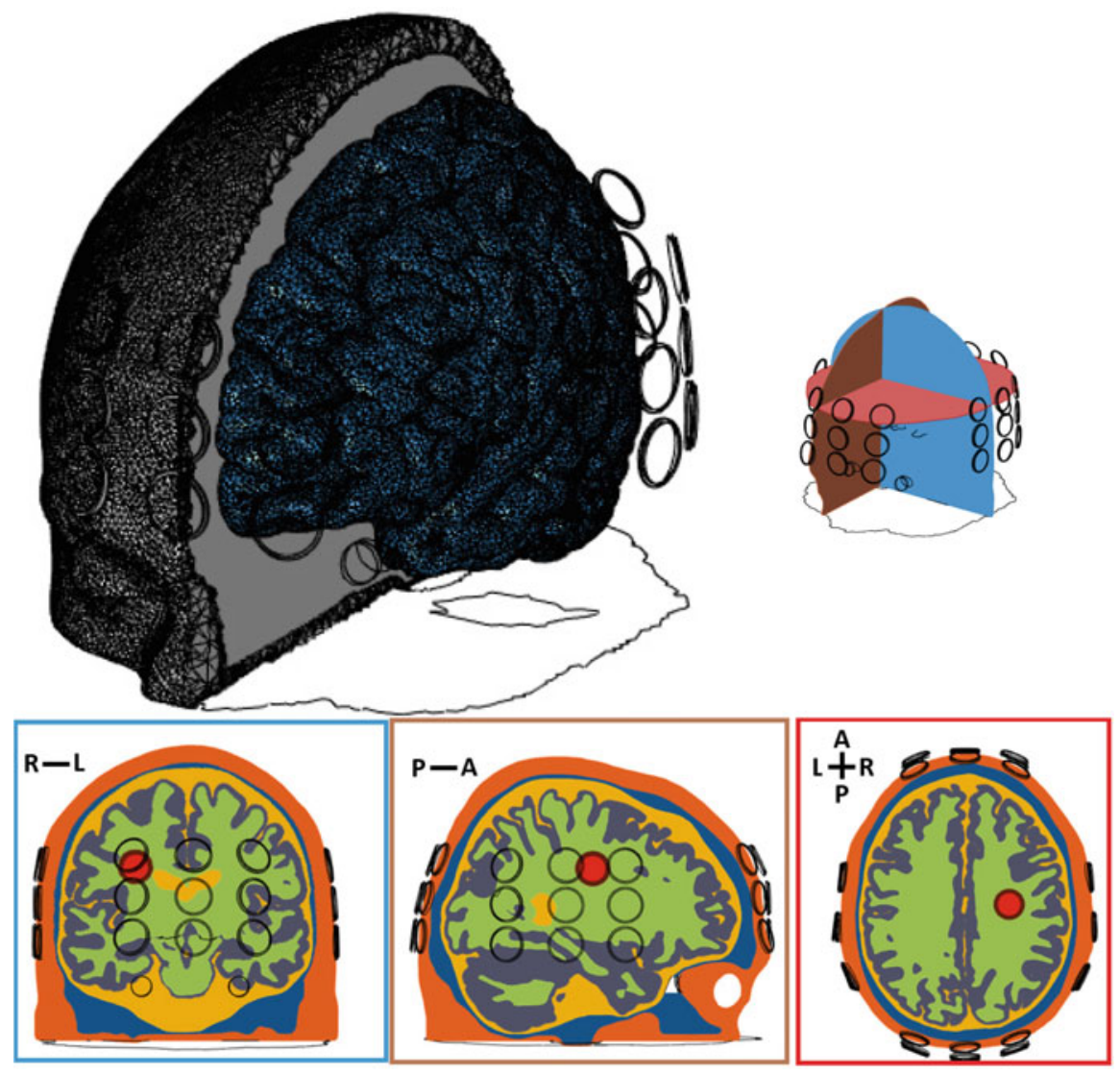

Fig. 1 Top: Partial representation of the mesh for the realistic human head model. Bottom: coronal (left), sagittal (middle), and axial (right) cuts through the center mass of the virtual lesion. Each color represents a different tissue: scalp (orange), skull (blue), CSF (yellow), gray matter (gray), white matter (green), tumor's outer shell (brown), and tumor's necrotic core (red). The eyes and the optical foramina are not colored

there are two main mechanisms that are responsible for increasing tissues' temperature: their metabolic activity and the Joule effect. As noted previously, the significance of the latter is a consequence of the high daily compliance needed for this therapy. The possible thermal harm is thus predicted by considering all these mechanisms and evaluating their relative contribution to the temperature distribution.

\subsubsection{Conduction}

Thermal conduction can be seen as a process that involves atomic and molecular motion [21]. When there is a temperature difference between two bodies in contact, 
heat will flow from the most energetic particles to the less energetic ones through vibration and collision. The rate equation that characterizes this process is known as Fourier's law:

$$
F=-k \nabla T
$$

where $F\left(\mathrm{~W} / \mathrm{m}^{2}\right)$ is the heat flux, $k\left(\mathrm{~W} /\left(\mathrm{m} \cdot{ }^{\circ} \mathrm{C}\right)\right)$ the thermal conductivity, and $T\left({ }^{\circ} \mathrm{C}\right)$ the temperature. This is the main process through which energy flows from the most internal tissues to the most superficial ones. For the head tissues we considered, the lowest thermal conductivity is scalp's $\left(0.34 \mathrm{~W} /\left(\mathrm{m} \cdot{ }^{\circ} \mathrm{C}\right)\right)$, and the highest is skull's $\left(1.16 \mathrm{~W} /\left(\mathrm{m} \cdot{ }^{\circ} \mathrm{C}\right)\right)$.

\subsubsection{Convection}

Heat transfer through convection occurs when a fluid in motion is in contact with a bounding surface that is at a different temperature. This mechanism is a result of the contribution of two different processes [21]: random molecular motion (i.e., diffusion) and bulk motion of the fluid (advection). The rate equation that describes this phenomenon is:

$$
F=h\left(T_{\text {surface }}-T_{\text {fluid }}\right)
$$

where $h\left(\mathrm{~W} /\left(\mathrm{m}^{2} \cdot{ }^{\circ} \mathrm{C}\right)\right)$ is the convection coefficient and $T_{\text {surface }}$ and $T_{\text {fluid }}$ are the bounding surface and fluid temperatures $\left({ }^{\circ} \mathrm{C}\right)$, respectively. This mechanism can decrease the temperature of the scalp, transducers, and gel as they are in contact with the cooler air. We considered $h=4\left(\mathrm{~W} /\left(\mathrm{m}^{2} \cdot{ }^{\circ} \mathrm{C}\right)\right)$ for the scalp and $h=0.25\left(\mathrm{~W} /\left(\mathrm{m}^{2} \cdot{ }^{\circ} \mathrm{C}\right)\right)$ for the medical tape covering the arrays. There are two other regions where convection can occur: between the blood and each perfused tissue, which is discussed below, and between the CSF and adjacent tissues (GM and skull). Heat exchange between the brain and CSF is still under investigation [22]. The very large surface contact between these two tissues plays an important role in maintaining brain's thermal safety as well as in providing structural and biochemical support [23]. Nonetheless, diffusion of molecules between the brain and the CSF is very restrained [24], and there is barely any study that describes the role of CSF advection in brain's temperature control. Further studies are also needed to characterize the importance of convection between skull and CSF.

\subsubsection{Radiation}

Heat transfer through radiation occurs whenever a body is at a nonzero temperature. For a given room temperature, the net radiation loss, in $\mathrm{W} / \mathrm{m}^{2}$, is given by: 


$$
F=\varepsilon \sigma\left(T_{\text {surface }}^{4}-T_{\text {room }}^{4}\right)
$$

where $\varepsilon$ represents the surface emissivity (unitless), $\sigma$ is the Stefan-Boltzmann constant $\left(5.67 \times 10^{-8} \mathrm{~W} /\left(\mathrm{m}^{2} \cdot \mathrm{K}^{4}\right)\right)$, and $T$ are the temperatures in $K$. According to Wien's displacement law, loss of energy through radiation at body temperature falls within the infrared region. Radiation is significant for all surfaces exposed to the environment (scalp, gel, and transducers).

\subsubsection{Sweat}

In hot environments when ambient temperature is higher than the skin's temperature, sweat is the only way for the body to dissipate heat [20]. Under normal conditions, sweat rate is around $600-700 \mathrm{~mL}$ per day [19], though this value can change depending on the environment, physical activity, and physiological conditions.

An equation that could realistically model sweat losses has been sought for a long time. The first attempts could not satisfactorily predict these losses for a practical range of conditions, either environmental or considering all the remaining significant energy processes. Shapiro's equation [25] is typically considered to be the first mathematical expression to correctly model whole body sweat losses in response to exercise, environment, and clothing. In their study, 34 heat-acclimatized subjects walked in a treadmill in different environments and wearing distinct outfits. The data obtained allowed the authors to deduce the following equation for sweat losses:

$$
\text { Sweating rate }\left(\mathrm{W} / \mathrm{m}^{2}\right)=18.7 \times E_{\text {req }} \times\left(E_{\max }\right)^{-0.455}
$$

where $E_{\mathrm{req}}\left(\mathrm{W} / \mathrm{m}^{2}\right)$ is the required evaporative cooling necessary to maintain thermal balance and $E_{\max }\left(\mathrm{W} / \mathrm{m}^{2}\right)$ is the maximum evaporative capacity of the environment. Both these quantities are defined in [26]. For a cooling effect to occur, $E_{\max }$ should be higher than $E_{\text {req, }}$, which is normally the case. In some situations, when air vapor pressure is too high, such as in humid conditions, an increase in the skin vapor pressure and wetted area is not enough to cool down the body, and thus heat storage occurs. Mathematically, $E_{\text {req }}$ is defined as:

$$
E_{\text {req }}\left(\mathrm{W} / \mathrm{m}^{2}\right)=\left[M_{\text {net }}+(R+C)\right] / 1.8
$$

$M_{\text {net }}(W)$ is the metabolic heat load given by the difference between body's total metabolic rate $(M$, in $W)$ and the external work performed by the subject walking in a treadmill. The factor 1.8 represents the average surface of the human body (in $\mathrm{m}^{2}$ ).

$$
M_{\text {net }}(W)=M-\left(0.098 m_{t} \times v \times G\right)
$$


In the previous equation, $m_{t}$ is the mass of the subject (in $\mathrm{kg}$ ), $v$ the walking speed (in $\mathrm{m} / \mathrm{s}$ ), and $G$ the grade (in $\%$ ) of the treadmill during the experiments. The value 0.098 is a conversion factor (in $\mathrm{m} / \mathrm{s}$ ). The authors calculated the metabolic rate as:

$$
M(W)=m_{t}\left[\left(2.7+3.2(v-0.7)^{1.65}\right)+G(0.23+0.29(v-0.7))\right]
$$

At rest (i.e., when $v=0 \mathrm{~m} / \mathrm{s}$ ), $M$ is assumed to be $105 \mathrm{~W}$.

The environmental heat load due to radiation and convection $(R+C)$, in $W$, is defined as:

$$
(R+C)(W)=\left(\frac{11.6}{\text { clo }}\right)\left(T_{\text {air }}-T_{\text {skin }}\right)
$$

In the former expression, clo represents the total thermal resistance of subject's clothes, which was a factor of interest in this experiment. A value of zero would correspond to a naked person, whereas clo $=1$ is the insulating value of clothing needed to maintain a person at rest in comfort in a room at $21^{\circ} \mathrm{C}$, with air movement of $0.1 \mathrm{~m} / \mathrm{s}$ and humidity less than $50 \%$. Typical values for this parameter and for different outfits can be found in [27].

On the other hand, $E_{\max }$ in Eq. (4) is given by:

$$
E_{\max }\left(\mathrm{W} / \mathrm{m}^{2}\right)=\frac{25.5}{1.8}\left(\frac{i_{m}}{\text { clo }}\right)\left(P_{\text {skin }}-P_{\text {air }}\right)
$$

$P_{\text {skin }}$ and $P_{\text {air }}$ are the vapor pressure in the skin and air (in $\mathrm{mmHg}$ ), respectively. Similarly to the clo factor, the permeability index of clothing, $i_{m}$, also depends on the subject's clothes [26]. A value of zero means that the fabric is impermeable, whereas a value of 1 means that all the moisture passes through the material.

The equation for sweat deduced by Shapiro and colleagues is valid for ambient temperatures between 20 and $54{ }^{\circ} \mathrm{C}$ and a relative humidity between $10 \%$ and $90 \%$. Furthermore, $E_{\text {req }}$ is limited to a range of values between 50 and $360 \mathrm{~W} / \mathrm{m}^{2}$, while $E_{\max }$ range of validity is between 20 and $525 \mathrm{~W} / \mathrm{m}^{2}$. This equation was largely used during more than 20 years to predict sweat losses. However, a more recent study [28] showed that it might be overestimating the predicted values. Additional experimental tests were made for a wider range of conditions, and a correction equation was deduced by Gonzalez et al. [28]:

$$
\text { Corrected Shapiro's equation }\left(\mathrm{g} /\left(\mathrm{m}^{2} \cdot \mathrm{h}\right)\right)=147 \exp (0.0012 \times \mathrm{OSE})
$$

where $h$ means hours and OSE stands for original Shapiro equation (in $\mathrm{g} /\left(\mathrm{m}^{2} \cdot \mathrm{h}\right)$ ). To convert the result to $\mathrm{W} / \mathrm{m}^{2}$, it is necessary to multiply the value obtained from the previous equation by water's latent heat $\left(2410 \mathrm{~J} / \mathrm{g}\right.$ at $\left.37^{\circ} \mathrm{C}\right)$ and by $1 / 3600 \mathrm{~h} / \mathrm{s}$. This correction proved to increase the accuracy of the results by $58 \%$. Additionally, a new sweat equation was also purposed: 


$$
\begin{aligned}
& \text { Gonzalez's sweating equation }\left(\mathrm{g} /\left(\mathrm{m}^{2} \cdot \mathrm{h}\right)\right) \\
& =147+1.527 \times E_{\mathrm{req}}-0.87 \times E_{\max }
\end{aligned}
$$

The last equation proved to be even more accurate $(65 \%)$ in predicting sweat losses. The last two equations are valid for ambient temperatures between 15 and $46{ }^{\circ} \mathrm{C}$, water vapor pressure between 0.27 and $4.45 \mathrm{kPa}$, and wind speed of $0.4-2.5 \mathrm{~m} / \mathrm{s}$.

Occurrence of sweat in TTFields patients is not an atypical situation [16]. It is observed when the environment is hot and humid or when the patient wears a wig to cover the arrays. Sweat represents an additional way to cool down the head. Although the number of eccrine glands varies depending on the region of the scalp, it is commonly believed that the forehead has a higher density of glands [29]. Thus, this is also the region that might cool down the most when sweating occurs. However, due to other aspects discussed further ahead, not all sweat is directly transferred to the environment by evaporation.

\subsubsection{Metabolism}

Metabolism can be defined as the necessary chemical reactions that occur in cells for the body to maintain its function and respond to external stimuli. As a result of this process, heat is released. The sum of the basal metabolic rate of cells with the rate from physical exercise and extra chemical reactions results in the final metabolic rate of the body [19]. For head tissues in particular, this mechanism can contribute significantly to temperature increases due to the brain's activity, which is a highly metabolic demanding organ. It accounts for $25 \%$ of the body's total glucose usage and around $20 \%$ of the total oxygen consumption [23]. Not surprisingly, in our model, the GM had the highest metabolic rate $\left(16,229 \mathrm{~W} / \mathrm{m}^{3}\right)$, whereas the CSF had the lowest $\left(0 \mathrm{~W} / \mathrm{m}^{3}\right)$.

\subsubsection{Blood Perfusion}

When body's temperature is too high, vasodilation of blood vessels is one of the main mechanisms to dissipate heat along with sweating and a decrease in the heat production [19]. For instance, vasodilation can increase heat exchange through the skin by a factor of 8 [19]. As previously mentioned, the physical process behind this cooling mechanism is convection between the blood and perfused tissues. A mathematical expression was deduced by Pennes [30] to express blood perfusion for each tissue:

$$
\text { Blood perfusion }\left(\mathrm{W} / \mathrm{m}^{3}\right)=\omega_{b} \rho_{b} c_{b}\left(T_{b}-T\right)
$$


where $\omega$ is tissue's blood perfusion rate $\left(\mathrm{s}^{-1}\right), \rho$ is the density $\left(\mathrm{kg} / \mathrm{m}^{3}\right), c$ the specific heat $\left(\mathrm{J} /\left(\mathrm{kg} \cdot{ }^{\circ} \mathrm{C}\right)\right)$, and $T$ the temperature $\left({ }^{\circ} \mathrm{C}\right)$. The subscript $b$ stands for blood. As the brain is the organ that needs more energy, it is also the one which has the highest blood perfusion. We considered GM and WM's perfusion rate to be $0.0133 \mathrm{~s}^{-1}$ and $0.0037 \mathrm{~s}^{-1}$, respectively.

\subsubsection{Joule Heating}

Joule heating, also known as Ohmic heating, occurs when there is a potential difference between two regions of a conductive medium. Electrons collide with the surroundings and energy transfer in the form of heat occurs. Mathematically, this term can be expressed as:

$$
\text { Joule heating }\left(\mathrm{W} / \mathrm{m}^{3}\right)=\boldsymbol{J} \cdot \boldsymbol{E}=\sigma\|\boldsymbol{E}\|^{2}
$$

where $\boldsymbol{J}$ is the current density vector $\left(\mathrm{A} / \mathrm{m}^{2}\right), \boldsymbol{E}$ is the electric field vector $(\mathrm{V} / \mathrm{m})$, and $\sigma$ is the electric conductivity $(\mathrm{S} / \mathrm{m})$. In TTFields the need to maximize the EF intensity at the tumor bed and the time that the patient is subjected to the field lead inevitably to temperature increases which may have a significant impact on tissues.

\subsection{Heat Transfer in TTFields: Pennes' Equation}

Pennes' equation [30] was used to obtain the temperature distribution as a function of space and time. This equation was derived based on experimental measurements of the temperature on the human forearm, and it is defined as:

$$
\rho c \frac{\partial T}{\partial t}=\underbrace{\nabla \cdot(k \nabla T)}_{\text {Conduction }}+\underbrace{\omega_{b} \rho_{b} c_{b}\left(T_{b}-T\right)}_{\text {Blood perfusion }}+\underbrace{Q_{m}}_{\text {Metabolic heat }}+\underbrace{\boldsymbol{J} \cdot \boldsymbol{E}}_{\text {Joule heating }}
$$

Some of the heat transfer mechanisms previously discussed are already considered in this equation. The remaining heat processes (convection, radiation, and sweat) were considered at the boundaries. More specifically, we considered energy exchanges through radiation and convection to occur on the outer boundaries of the scalp, transducers, and gel.

In our model, the medical tape that covers the arrays is not represented. In a real patient, this adhesive covers a large part of the scalp, and thus it changes the rate at which energy is exchanged with the environment. To minimize the error associated with this lack of representation, we chose emissivity and convection factor values for the transducers and the gel that were close to that of the medical tape. As previously mentioned, TTFields patients might sweat to reduce the temperature increases that 
occur on the scalp, but some of this energy might not be transferred to the environment. The fabric of the medical tape can retain some of the sweat, and consequently changes in the hydrogel composition could occur. Due to all these uncertainties and the fact that there is no equation that predicts cooling by sweating just for the head, we opted to use a constant value based on Gonzalez's sweat equation for the whole scalp $\left(125 \mathrm{~W} / \mathrm{m}^{2}\right)$ except where covered by the gel and transducers.

\subsection{Simulations' Conditions}

The frequency used in TTFields therapy, $200 \mathrm{kHz}$, falls within the frequency range where the electromagnetic wavelength is significantly larger than the size of the human head which allowed us to use the electroquasistatic approximation of Maxwell's equations. Under this approximation, the electric potential could be calculated using Laplace's equation. The AC/DC module (electric currents, frequency domain study) was used in COMSOL Multiphysics to obtain the electric field distribution when the AP configuration was on. The potential difference between the anterior and posterior arrays $\left(V_{\mathrm{AP}}\right)$ was defined in such a way that a current with an amplitude of $900 \mathrm{~mA}$ passed between the two arrays. In our model, this corresponded to having $V_{\mathrm{AP}}=91.8 \mathrm{~V}$. This study took around $8 \mathrm{~h}$ in a workstation with $2 \times 4$ core CPU's (Intel Xeon W5580@ 3.2 GHz) and 48 GB RAM. Solver's relative tolerance was set to $10^{-6}$. At the end of this simulation, we obtained a total power density spatial map that represented the contribution of this configuration to the Joule effect.

The Heat Transfer Module (electromagnetic heating, Joule heating, frequencytransient study) was then used to calculate the electric field distribution when the LR configuration was applied and also to predict the temperature increases. Similarly to the previous study, a fixed potential difference was defined between the left and right arrays $\left(V_{\mathrm{LR}}=68.2 \mathrm{~V}\right)$ to ensure that $900 \mathrm{~mA}$ were injected.

Regarding the Joule heating term, the last term of Eq. (14), the contribution from the AP and LR configurations was taken alternately every second after both studies were coupled. Each simulation of $360 \mathrm{~s}$ took around $22 \mathrm{~h}$ in the aforementioned workstation. We also assumed that all the physical properties were isotropic and uniform. The values assigned to each tissue and material were chosen after an extensive literature review. Additional details about these values and how the studies were performed can be found in [31].

\section{Results}

\subsection{Duty Cycle and Effective Electric Field at the Tumor}

In our first work [31], we predicted the thermal impact of TTFields and quantified the duty cycle of Optune. We defined the latter as the time that each configuration is on 


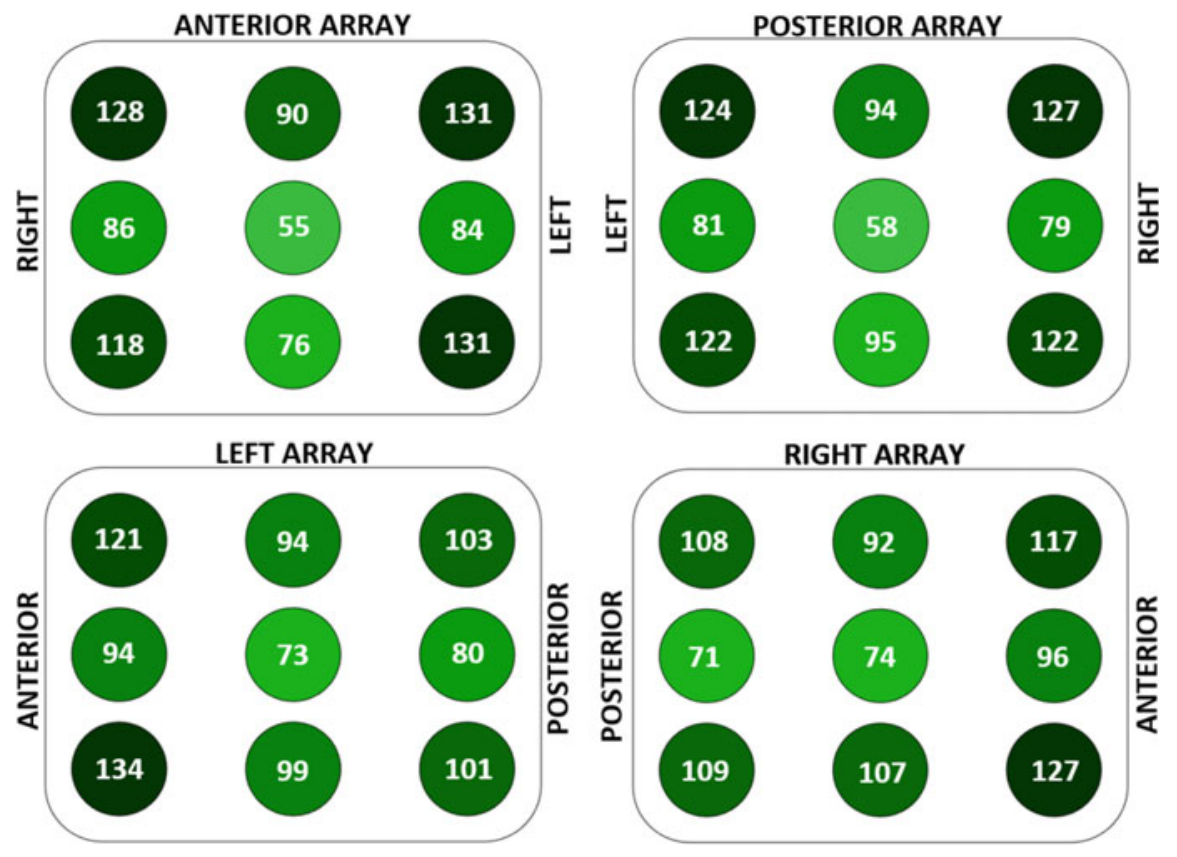

Fig. 2 Absolute current injected in each transducer. Each array can be seen as an isopotential surface where the edge effects are evident as more current is injected in the outer transducers. All values are in $\mathrm{mA}$

divided by the maximum time it could be delivering the fields and assumed that there was a complete current shutdown whenever the average temperature of any transducer reached a cutoff temperature. We considered this critical value to be $40.4{ }^{\circ} \mathrm{C}$ because it corresponded to having around $5 \%$ of the volume of the transducer at $41{ }^{\circ} \mathrm{C}$, which we assumed to be the volume occupied by the temperature sensor. In this work, we did not consider heat losses through sweat. Our simulations showed that there was one transducer whose temperature reached the threshold temperature quicker than the other 35 (the Most Significant Transducer or MST). Consequently, it was the one that controlled if current was injected or not. In our head model in particular, it was the transducer located in the anterior array, superior row, left side (ASL). Not surprisingly, it was also one of the transducers where the most current was injected (around $131 \mathrm{~mA}$, see Fig. 2). Each array can be seen an isopotential surface in which more current is injected through the outer transducers, a phenomenon commonly known as the edge effect [32].

According to our results, Optune's way of operating can be summarized as follows: at the beginning of treatment, current is injected in two perpendicular directions alternately. Transducers' temperature starts to increase at different rates and biological tissues' temperature also rises. After around two and a half minutes, the temperature of one transducer (the MST) exceeds the safety threshold of $40.4^{\circ} \mathrm{C}$, and the fields are completely shut down in both directions. It then takes around 2-4 s 


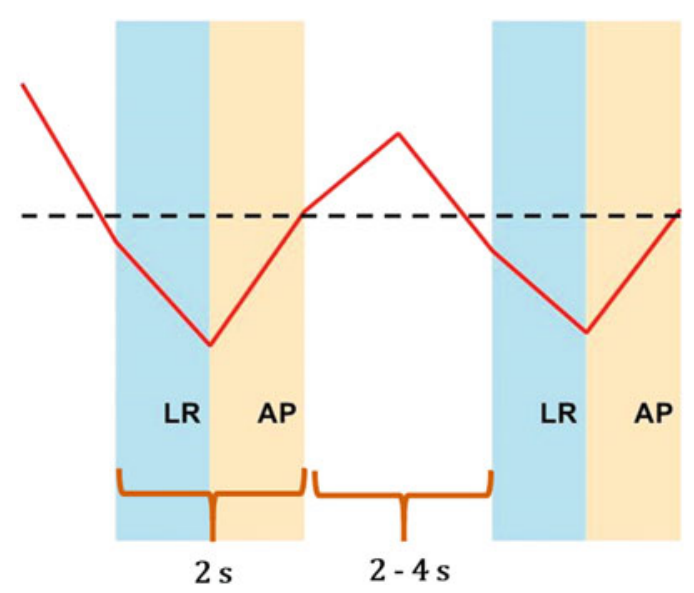

Fig. 3 Temperature variation of the Most Significant Transducer during intermittent operation. The dashed line represents the critical temperature $\left(40.4{ }^{\circ} \mathrm{C}\right)$. After each shutdown, it takes around $2-4 \mathrm{~s}$ for the temperature of the transducer to decrease below the $40.4{ }^{\circ} \mathrm{C}$. After current starts to be applied again, it takes about $2 \mathrm{~s}$ to surpass once again the threshold. These heating and cooling rates lead to different duty cycles for each configuration

to decrease the temperature of the MST to values below the critical limit and consequently to start injecting current again. After $2 \mathrm{~s}$ of alternate application of the fields, the temperature will once again surpass the threshold, and current shutdown will occur once more. This represents an intermittent operating mode of Optune (Fig. 3).

This led to different duty cycles for each configuration. In our model, current was injected in the AP direction $47 \%$ of the maximum time, while this value decreased to around $30 \%$ for the LR configuration. This means that, on average, Optune is used for GBM treatment around $39 \%$ of the time that the patient is using the device.

To know the effective electric field in the tumor, we started by calculating the EF magnitude induced by each configuration in this region. We defined ATV1 as the percentage of the tumor shell volume above the therapeutic threshold of $1 \mathrm{~V} / \mathrm{cm}$. As it can be seen in Fig. 1, the tumor is much closer to the right array and more or less at the same distance to the anterior and posterior arrays. This explains why the ATV1 value for the AP configuration is just $13 \%$, while for the LR is $72 \%$. Following this rationale, we can define the effective ATV1 (ATV1 $\left.1_{\text {eff }}\right)$ at the tumor shell as the combined contribution of the duty cycle and the induced electric field as:

$$
\begin{aligned}
\mathrm{ATV} 1_{\mathrm{eff}}^{\text {std }} & =\mathrm{On}_{\mathrm{AP}} \times \% \mathrm{ATV} 1_{\mathrm{AP}}+\mathrm{On}_{\mathrm{LR}} \times \% \mathrm{ATV} 1_{\mathrm{LR}} \\
& =0.47 \times 13 \%+0.30 \times 72 \%=27.7 \%
\end{aligned}
$$

where $\mathrm{On}_{x}$ is the fraction of time that configuration $x$ is on and ATV $1_{x}$ the percentage of the tumor volume above $1 \mathrm{~V} / \mathrm{cm}$ when this configuration is applied. 


\subsection{Improving the Duty Cycle}

There are two possible ways to increase the effective ATV1: by rising the electric field magnitude at the tumor shell or by increasing the duty cycle. We investigated these possibilities by performing different studies (studies 1-4) where we slightly changed how Optune worked. Here we discuss the operating modes that improved the results. Note that these results do not consider heat losses through sweat. For more details, see [31].

We first hypothesized that decreasing the amount of injected current by a factor of $1 / \sqrt{ } 2$, but activating both arrays simultaneously, might allow to reduce the temperature maxima by reducing the Joule effect by half and thus increasing the time that the device is on (study 1). This operating mode affected both the electric field magnitude and direction and resulted in a duty cycle of $70 \%$. However, the ATV1 decreased to around $45 \%$ of the tumor shell. Even so, this results in an effective ATV1 of $31.5 \%$, which is more than the $27.7 \%$ observed in the standard configuration. Additionally, in this mode, current is injected only in one direction because the EF is a vector quantity, and thus the net field is oriented at approximately $45^{\circ}$ relative to the standard AP and LR directions. However, it is possible to intentionally change the phase of the injected current so that more electric field directions are achieved. The latter approach might increase the number of affected cells and thus enhance treatment outcome [1].

We then changed the switching time between configurations. Currently, Optune alternates the direction of the applied field every second as this is the optimal time to affect cell division according to in vitro studies. We investigated the impact of increasing the switching time to $2 \mathrm{~s}$ (study 2). Our results showed that although this leads to higher temperatures, the cooling rate is also augmented because it depends on the temperature difference with the environment. As a consequence, the duty cycle improved for this case, reaching $47 \%$ and $40 \%$ for the AP and LR configurations, respectively. Thus, the effective ATV1 also increased to $34.9 \%$. It is important to note that switching times different from $1 \mathrm{~s}$ might not be the most suitable to affect the mitotic process of GBM cells. For instance, a recent in vitro study of Berkelmann et al. [21] concluded that for the BT4Ca cell line, the optimal switching time that maximizes the antiproliferative effect of TTFields is $60 \mathrm{~s}$.

In all the studies we performed, there was always one transducer that controlled if Optune was injecting current or not. Due to the cooling time and the location of the MST on the anterior array, the LR configuration was applied less time than the AP's. We hypothesized that if temperature control was made independently for each configuration, then the duty cycle would increase. To apply this feature, we considered that there could be two MST's, one for each configuration. In practice, this means that if a transducer of one configuration reaches the threshold temperature $\left(40.4{ }^{\circ} \mathrm{C}\right.$ ), we would only shut down that specific configuration, while the other could continue to operate alternately until a transducer of that configuration reached the critical temperature (Study 3). In these conditions, the duty cycle for the AP 
Fig. 4 Effective volume of the tumor shell above $1 \mathrm{~V} /$ $\mathrm{cm}$. Study 1: less current injected, but all arrays were simultaneously activated. Study 2: switching time between configurations increased to $2 \mathrm{~s}$. Study 3 : temperature control for each configuration is made independently. Study 4: each transducer gets exactly $100 \mathrm{~mA}$

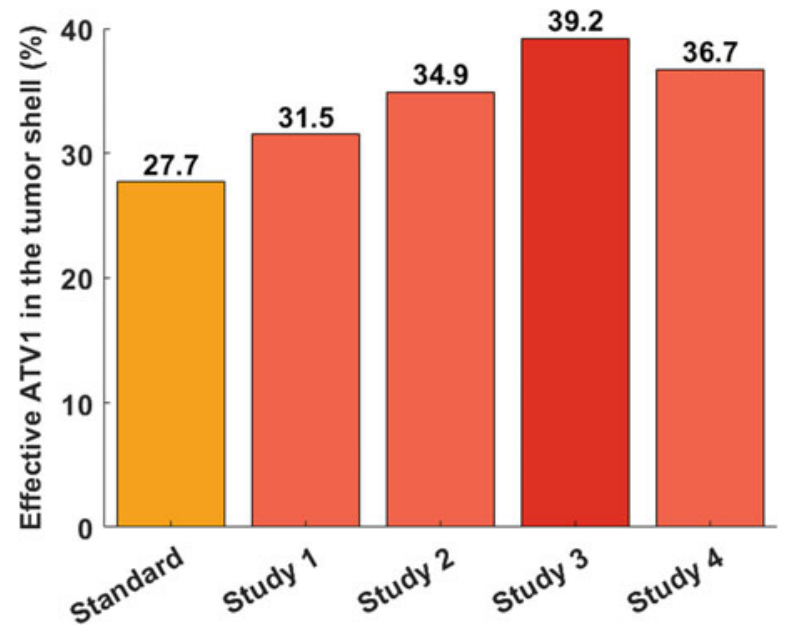

configuration was $47 \%$, while current was injected in the LR direction $46 \%$ of the time, leading to an ATV1 effective of $39.2 \%$.

Lastly, we tried to increase the electric field in the tumor. We investigated what would happen if we avoided the edge effects by injecting exactly $100 \mathrm{~mA}$ in each transducer (Study 4). We noticed that this changed the MST and also that it improved the duty cycle (AP: 46\%; LR: 42\%), although the ATV1 at the tumor bed did not change significantly compared to the standard operation mode. In this case, the ATV1 $1_{\text {eff }}^{\text {std }}$ increased to $36.7 \%$.

The effective ATV1 for each study is presented in Fig. 4.

Our results point out that the best way to improve TTFields therapy, among the hypotheses we tested, is by controlling the current into the array pairs independently but still alternately. This allows to compensate the fact that, in our model, there was always one transducer that controlled if the device was applying the fields or not. Including this feature in Optune might imply changing the device's hardware and software.

As the duty cycle increases, so does the Joule effect, which can lead to significant temperature increases compared to the standard case. In Sects. 3.4 and 3.5, we analyze temperature changes and make predictions about the thermal impact of this technique.

\subsection{The Effect of Sweat}

Before predicting the thermal impact, we started by studying the main differences in the temperature distributions and duty cycle if we considered heat losses through sweat. As already stated, we considered a constant value of $125 \mathrm{~W} / \mathrm{m}^{2}$ for the whole scalp except at the regions where the transducers were placed. In these conditions, 
the cooling time decreased, and it took around $2 \mathrm{~s}$ to cool down the MST to temperatures below $40.4{ }^{\circ} \mathrm{C}$. This led to a duty cycle of around $50 \%$ for both the $\mathrm{AP}$ and LR configurations.

The effective ATV1 considering sweat is then:

$$
\begin{aligned}
\mathrm{ATV} 1_{\mathrm{eff}}^{\text {sweat }} & =\mathrm{On}_{\mathrm{AP}} \times \% \mathrm{ATV} 1_{\mathrm{AP}}+\mathrm{On}_{\mathrm{LR}} \times \% \mathrm{ATV} 1_{\mathrm{LR}} \\
& =0.50 \times 13 \%+0.50 \times 72 \%=42.5 \%
\end{aligned}
$$

Comparing the latter value with the ones presented in Fig. 4, it is possible to conclude that adding sweat to the model has led to the largest improvement in the predicted outcome for this therapy. Given the importance of this cooling mechanism, it would be important to improve and validate the model used. Additionally, it would also be interesting to add sweat losses and combine the different conditions represented by studies $1-4$ and quantify the duty cycle.

\subsection{Temperature Increases}

The temperature distribution on each tissue surface for the standard operating mode and without considering sweat can be seen in Fig. 5. The temperature distribution on each tissue surface with this additional cooling mechanism is shown in Fig. 6.

The two figures clearly show localized temperature increases, mainly underneath the regions where the transducers were placed. In Fig. 5, temperature on the left and right sides is lower compared to the values presented in Fig. 6 because the duty cycle for the LR configuration is also lower. Even though the duty cycle was increased to nearly $50 \%$ for both configurations when sweat was considered, the maximum temperatures reached by each tissue were practically the same in both situations because current shutdown occurred at the same temperature. Given that there are electric field hotspots at tissue interfaces due to accumulation of charges [18], the Joule effect will also be more significant in these regions. Consequently, it is expected that the temperature maxima in each tissue occur on the surfaces. In Fig. 7 this superficial heating is well seen on the brain's surface. Despite the fact that both configurations are applied for the same time, the temperature increases are higher in the anterior-posterior direction as it is clearly shown in Fig. 6. This is a result of the array positioning in our model. The distance between the central transducers of the arrays pairs is around $20 \mathrm{~cm}$ for the AP configuration and $17 \mathrm{~cm}$ for the LR. Under these circumstances, the potential difference between each pair is higher for the first to ensure that $900 \mathrm{~mA}$ are injected (91.8 V vs. $68.2 \mathrm{~V}$, both values are in amplitude), and thus the electric field and Joule heating will also be higher near this array pair. This explains why the MST is located in the anterior array. For other head model and another array layout, the most significant transducer might not be the same. 


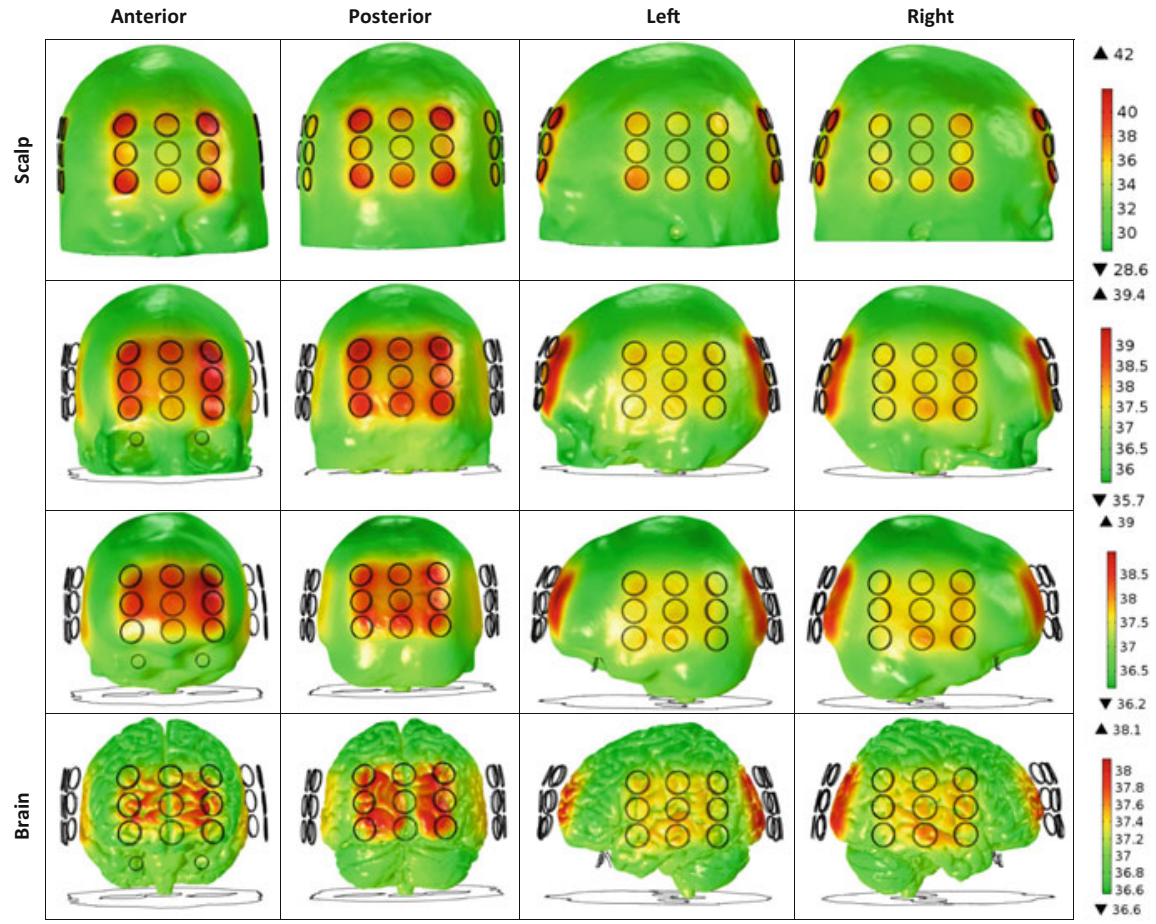

Fig. 5 Temperature distribution for Optune's standard operating mode without considering sweat and for each tissue surface at the end of the simulation (360 s). First row, scalp; second row, skull; third row, CSF; fourth row, the brain. Note the different scales for each row. All values are in ${ }^{\circ} \mathrm{C}$

\subsection{Prediction of the Thermal Impact}

We used two different metrics to predict the thermal impact of TTFields: the maximum temperature reached by each tissue and the cumulative number of equivalent minutes at $43{ }^{\circ} \mathrm{C}\left(\mathrm{CEM} 43{ }^{\circ} \mathrm{C}\right)$. The latter was defined by Sapareto and Dewey [33] as:

$$
\mathrm{CEM} 43^{\circ} \mathrm{C}=\sum_{i=1}^{n} t_{i} R^{43-T_{i}}
$$

In this equation, $t_{i}(\mathrm{~s})$ and $T_{i}\left({ }^{\circ} \mathrm{C}\right)$ are the duration of the $i$-th time interval and temperature during that period, respectively, $n$ is the total number of intervals, and $\mathrm{R}$ is a constant related with the thermotolerance acquired by the cells. It has a value of 0.5 for temperatures higher than $43^{\circ} \mathrm{C}$ and 0.25 otherwise. Thresholds for thermal effects based on CEM $43{ }^{\circ} \mathrm{C}$ values are available in the literature $[34,35]$.

Given that the temperature increases are very localized (Figs. 6 and 7) and occur mainly underneath the arrays, we chose to calculate the CEM $43{ }^{\circ} \mathrm{C}$ for each tissue 


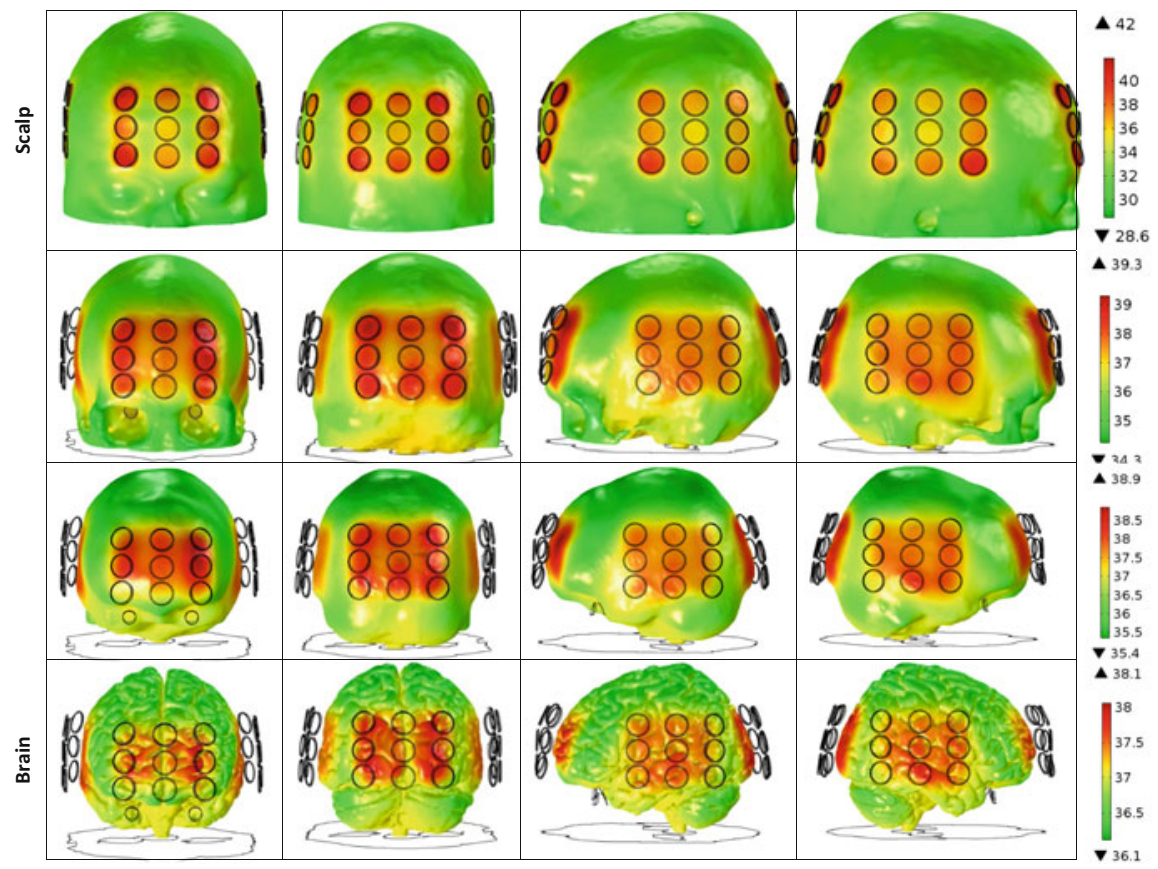

Fig. 6 Temperature distribution on each tissue surface at the end of the simulation ( $360 \mathrm{~s}$ ) for Optune's standard operating mode when sweat is considered for the scalp. First row, scalp; second row, skull; third row, CSF; fourth row, the brain. Note the different scales for each row. All values are in ${ }^{\circ} \mathrm{C}$

within a cylinder defined by the MST and for the results represented in Fig. 6. The basis of this cylinder has the area of the transducer, and the cylinder's axis is parallel to the transducer surface's normal. To make our predictions, we only considered thresholds for thermal impact for the five tissues that were represented in our model. Additionally, because we only simulated the first 6 min of therapy, we assumed that the temperature at the last time step would remain the same to calculate the CEM $43{ }^{\circ} \mathrm{C}$ for a typical treatment day $(18 \mathrm{~h})$. This approach is feasible because the temperature in each tissue had practically reached a steady-state distribution [31].

Our analysis shows that the CEM $43^{\circ} \mathrm{C}$ thresholds for the scalp and skull were not surpassed, and thus thermal damage at histopathological and functional levels is not expected when the patient is subjected to the action of the fields. Regarding thermal safety for CSF, there are no thresholds defined in $[34,35]$ to compare our measurements to. We observed maximum increases of around $1.8^{\circ} \mathrm{C}$ in this tissue. Given that CSF is in contact with the interstitial fluid (ISF) and that the latter helps to dissipate heat from the brain [36], the actual temperature increase might be even lower. For the brain, we obtained a local CEM $43{ }^{\circ} \mathrm{C}$ value of 0.30 min underneath the transducers assuming $18 \mathrm{~h}$ of treatment. Some studies [34, 35] reported that values higher than $0.03 \mathrm{~min}$ are enough to increase the permeability of the bloodbrain barrier (BBB). Other possible effects include changes in the cerebral blood 


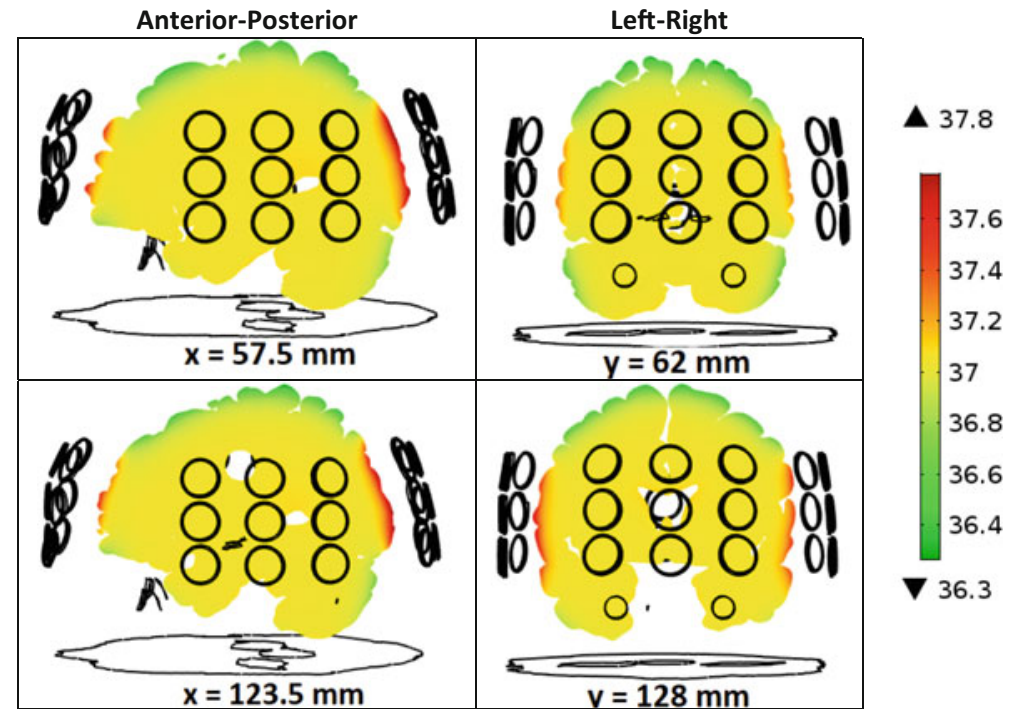

Fig. 7 Temperature distribution in the brain in four different slices under the arrays at $t=360 \mathrm{~s}$ when sweat is considered. Note that the temperature maxima occur at the surface under the transducers and that there is a quick drop to a constant value $\left(37^{\circ} \mathrm{C}\right)$. Values are in ${ }^{\circ} \mathrm{C}$

flow $(\mathrm{CBF})$ and variation in the concentration of some neurotransmitters. Regarding the last two, whether there is a rise or a decrease is not consensual among different studies. Some researchers observed a higher irrigation of cerebral tissue for CEM $43{ }^{\circ} \mathrm{C}$ values of 0.03 and $16 \mathrm{~min}$, whereas other showed a decreased blood flow for values around 1 and $34 \mathrm{~min}$. Neurotransmitter concentration was also seen to increase for values above $0.115 \mathrm{~min}$, but when CEM $43{ }^{\circ} \mathrm{C}$ surpassed $1.29 \mathrm{~min}$, there was a drop below the baseline concentration.

It is important to note, however, that there are a lot of uncertainties regarding the CEM $43{ }^{\circ} \mathrm{C}$ thresholds, and these conclusions might not hold when these limits are used to predict the thermal impact of tumor-treating fields. This metric was created with the intention of having a way to calculate a thermal isoeffect dose for cancer therapy. In other words, the rationale behind it is that it is possible to convert the time that a tissue is at a given temperature to an equivalent time at $43{ }^{\circ} \mathrm{C}$. However, this is not necessarily accurate. As pointed out by Sapareto and Dewey [33], the heating regime is an important parameter as well as when the damage is assessed, tissue's $\mathrm{pH}$, and the thermotolerance to heat of different cells. Not surprisingly, the lack of experimental protocols leads to different conclusions regarding variations of the same physiological process.

Due to all these uncertainties, it is first necessary to investigate if CEM $43{ }^{\circ} \mathrm{C}$ can be really applied to TTFields by measuring sensitive biomarkers to the changes here reported and confirm or disprove these hypotheses. In case that this metric proves to be a good measurement for thermal impact, specific thresholds for long and repetitive exposure times are needed. Most of the thresholds defined in the literature concern whole-body heating and were not specifically designed for the human head. 
Another way to predict thermal impact is by evaluating the maximum temperature reached by each tissue. The temperature maxima (see Fig. 6) are still well below $45{ }^{\circ} \mathrm{C}$, which is the temperature at which protein and lipid denaturation starts to occur [23]. Temperature on the scalp is also below the threshold of $44{ }^{\circ} \mathrm{C}$ for skin burn for long exposures, as reported by Moritz and Henriques [37]. In the brain, maximum temperature variations of around $2{ }^{\circ} \mathrm{C}$ were obtained. Studies performed in animals showed that the cerebral metabolic rate of oxygen $\left(\mathrm{CMRO}_{2}\right)[38,39]$ and CBF [39] increase more or less linearly with the temperature.

Volgushev et al. [40] also showed that temperature variations can temporarily change synaptic transmission in the neocortex. Further studies by the same author [41] revealed that there is an increase of the probability of neurotransmitter release at the synapses as the temperature increases.

Whether these changes can be harmful or not is not clear. An increase on the $\mathrm{CMRO}_{2}$ might lead to higher temperatures, but a higher CBF can also help to dissipate the heat more quickly. The uncertainty on the outcome is even higher when it comes up to changes at the synaptic level. The frequency used for TTFields treatment is too high to be capable of cell stimulation. Evidence from clinical trials $[6,8]$ shows no additional significant nervous system disorders when TTFields are used as a part of the standard of care which might indicate the absence or the low impact of these variations. As none of these effects were observed [6-8], it is necessary to understand why the theory does not match with the practice. The main explanations might be:

1. These changes do not occur during TTFields therapy: the conclusions drawn from the literature only apply to the conditions and technique for which those specific studies were performed. This implies that thresholds defined based on animal studies must be reviewed for humans.

2. These changes do occur but are negligible: given that the temperature variations are not very high, these physiological alterations are not meaningful, and thus they are not observed as side effects.

3. These changes occur but were not measured: the sensitive biomarkers for the changes here reported were not acquired.

4. These are long-term changes and are masked by patient's condition: these possible side effects might not occur on the first months of treatment, and only patients who survive longer manifest these alterations.

5. The high daily compliance leads to development of thermotolerance by the cells: given that the daily minimum recommended time for recurrent cases is $18 \mathrm{~h}$ and that treatment should be performed every day, cells might become less sensitive to temperature variations, and thus the thresholds should be higher in these conditions.

6. The predictions made are not correct: the model does not realistically represent an actual treatment, and consequently the temperature distribution is being overestimated.

The first step to exclude some of these hypotheses would be to acquire sensitive biomarkers during TTFields treatment. If these changes do occur, it is necessary to 
investigate further if they can be harmful or beneficial for the patient. On the other hand, if the predictions are not correct, further improvements should be made to our model to make it more reliable.

Regarding the tumor, the maximum temperature variation also occurred at the surface, although it was not higher than $0.1^{\circ} \mathrm{C}$. It is known that significant temperature increases can make cells more likely to be affected by radiation or some drugs, which explains why hyperthermia is sometimes used in cancer treatment. In our studies the maximum temperature reached by the tumor was $37.1^{\circ} \mathrm{C}$, which suggests that TTFields mechanisms of action are not expected to be temperature-related. However, a recent work by Berkelmann et al. [5] opened up new perspectives on the possible role of heat in this therapy. The authors predict a very high SAR value at the cleavage furrow during telophase and cytokinesis when the direction of the electric field is parallel to the longitudinal axis of the hourglass morphology of the dividing cells.

\subsection{Continuous Versus Intermittent Application of the Fields}

As seen in Sect. 3.1. due to the necessary thermal restrictions, Optune might work intermittently with duty cycle lower than $100 \%$. In our next study, we investigated by how much it was necessary to decrease the injected current so that the average temperature of the MST would not exceed the shutdown temperature $\left(40.4{ }^{\circ} \mathrm{C}\right)$. In other words, this means that if the injected current is below a critical value (the critical current or $I_{C}$ ), then it is possible to have an uninterrupted application of the fields and consequently a duty cycle of $100 \%$. Figure 8 shows the average temperature of the MST as a function of the injected current for a $360 \mathrm{~s}$ simulation when sweat is considered. Current was applied alternately between the two configurations with a switching time of $1 \mathrm{~s}$ and when no current shutdown is considered. Each simulation took around $24 \mathrm{~h}$ in the aforementioned workstation. An analytical function (Eq. 16) was fitted to each curve represented in this figure.

$$
T=C_{1}+C_{2} *\left(1-\exp \left(-t / C_{3}\right)\right)
$$

where $C_{1}\left({ }^{\circ} \mathrm{C}\right), C_{2}\left({ }^{\circ} \mathrm{C}\right)$, and $C_{3}(\mathrm{~s})$ are constants. The maximum average temperature $\left(T_{\max }\right.$, in ${ }^{\circ} \mathrm{C}$ ) reached by this transducer can be predicted by calculating the limit of $T$ when $t \rightarrow \infty$ :

$$
T_{\max }=C_{1}+C_{2}
$$

Table 1 summarizes $C_{1}, C_{2}$, and $C_{3}$ values, as well as $T_{\max }$, as a function of the injected current.

The physical meaning of each parameter is as follows: $C_{1}$ values represent the initial average temperature of the transducer; $C_{2}$ can be seen as the maximum contribution of the injected current to temperature increases. $C_{3}$ is a time constant 


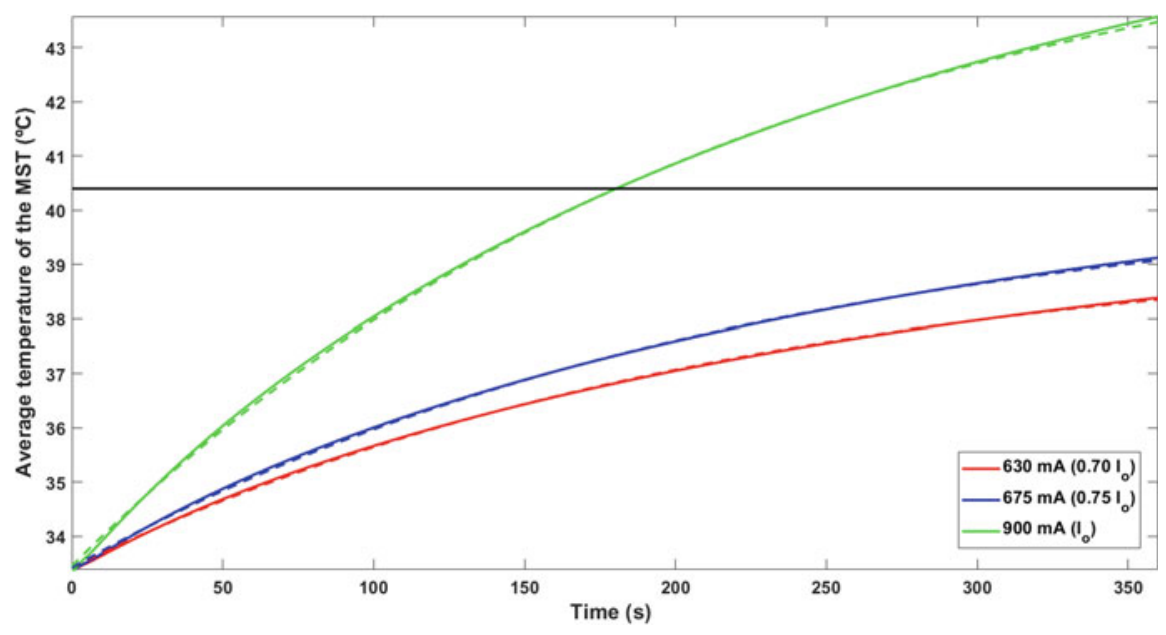

Fig. 8 Average temperature variation of the MST when no current shutdown is implemented. The dashed lines represent the best fit for each case based on the expression shown in Eq. (16)

Table 1 Curve fitting parameters as a function of the current injected into the MST

\begin{tabular}{l|l|l|l|l|l}
\hline$I(\mathrm{~mA})$ & $C_{1}\left({ }^{\circ} \mathrm{C}\right)$ & $C_{2}\left({ }^{\circ} \mathrm{C}\right)$ & $C_{3}(\mathrm{~s})$ & $R^{2}$ & $T_{\max }\left({ }^{\circ} \mathrm{C}\right)$ \\
\hline $900\left(\boldsymbol{I}_{0}\right)$ & 33.45 & 12.50 & 222.2 & 0.9998 & 45.95 \\
\hline $675\left(0.75 \boldsymbol{I}_{0}\right)$ & 33.42 & 7.05 & 222.2 & 0.9998 & 40.47 \\
\hline $630\left(0.70 \boldsymbol{I}_{0}\right)$ & 33.42 & 6.15 & 222.2 & 0.9998 & 39.57 \\
\hline
\end{tabular}

The values of R-squared suggest a high correlation between current and the average temperature reached by the MST

related with the heat transfer with the environment and the injected current. When $t=C_{3}$, the temperature of the MST reached around $63.2 \%$ of its maximum temperature. Figure 8 also shows in dashed lines the best curve fitting based on the parameters of Table 1 for each amount of injected current. Note that the fitted equation might underestimate the temperatures for long periods of time.

According to our calculations, the critical current is $674 \mathrm{~mA}$ which represents a decrease of around $25 \%$ compared to the current that is typically injected in a real patient. Naturally, this decrease is followed by a lower induced electric field in tissues and more specifically in the tumor. The ATV1 for each configuration in the tumor shell is given in Table 2 .

The ATV1 values for the AP configuration reduce to $0 \%$ for current values near $I_{C}$, which, as mentioned previously, has to do with tumor's location. In terms of treatment efficacy, decreasing the current to achieve a duty cycle of $100 \%$ leads to an effective ATV1 of:

$$
\mathrm{ATV} 1_{\text {eff }}^{\text {No_shut }}=0 \% \times 1.00+34 \times 1.00=34.0 \%
$$


Table 2 Percentage of the tumor shell volume above $1 \mathrm{~V} / \mathrm{cm}$ as a function of the injected current

\begin{tabular}{l|l|l}
\hline$I(\mathrm{~mA})$ & $\mathrm{ATV} 1_{\mathrm{AP}}(\%)$ & $\mathrm{ATV} 1_{\mathrm{LR}}(\%)$ \\
\hline $900\left(\boldsymbol{I}_{0}\right)$ & 13 & 72 \\
\hline $675\left(0.75 \boldsymbol{I}_{0}\right)$ & 0 & 39 \\
\hline $674\left(\boldsymbol{I}_{C}\right)$ & 0 & 34 \\
\hline $630\left(0.70 \boldsymbol{I}_{0}\right)$ & 0 & 31 \\
\hline
\end{tabular}

The latter value is still lower than the $42.5 \%$ obtained when current shutdown was implemented, which is mainly influenced by the AP configuration not producing a significant electric field (i.e., higher than $1 \mathrm{~V} / \mathrm{cm}$ ) at the tumor bed. Nonetheless, injecting less current to avoid the need of shutting down the fields might be a good solution to increase the effective ATV1 for more superficial tumors.

As expected, temperature maxima in the deepest tissues decreased for this new current injection mode because the power density dissipated in tissues was $44 \%$ lower compared to what was deposited when $900 \mathrm{~mA}$ were applied. The temperature distribution considering heat losses through sweat when $674 \mathrm{~mA}$ are injected continuously is shown in Fig. 9. Regardless of the injected current, the spatial distribution of the temperature on tissues' surface remains practically the same (compare Figs. 6 and 9). Despite the small decreases in CEM $43{ }^{\circ} \mathrm{C}$ and in the maximum temperature, the same physiological changes predicted in Sect. 3.5 apply to the results here presented.

\section{Limitations and Future Work}

One of the major drawbacks when modelling tumor-treating fields is the lack of a metric that allows to simultaneously evaluate the electric field, the duty cycle, and the temperature increases and to weigh the different parameters to find, e.g., the best array placement. Treatment efficiency is commonly quantified by metrics such as the ATV1 that allow to study if the induced EF is higher than the minimum therapeutic value or not. More recently, the power density has been used as a measurement of both the electric field and temperature increases [10]. In the future, it would be useful to develop and validate a new metric that combines all the features mentioned above.

The results of our simulations predict some effects that were not reported elsewhere. Acquiring the necessary biomarkers sensitive to the physiological changes here described would allow to corroborate or disprove our conclusions. If our model turns out to be inaccurate to represent a real patient and the conclusions derived from it are not reliable, then fine-tuning the most critical parameters is necessary. The latter includes having a more realistic equation to calculate sweat losses and perform a sensitivity analysis to evaluate the impact of the uncertainty of the electric and thermal parameters of tissues in the temperature distribution. Representation of the medical tape that covers the transducers, gel, and a large surface of the scalp would also make our model more realistic. Additionally, performing this 


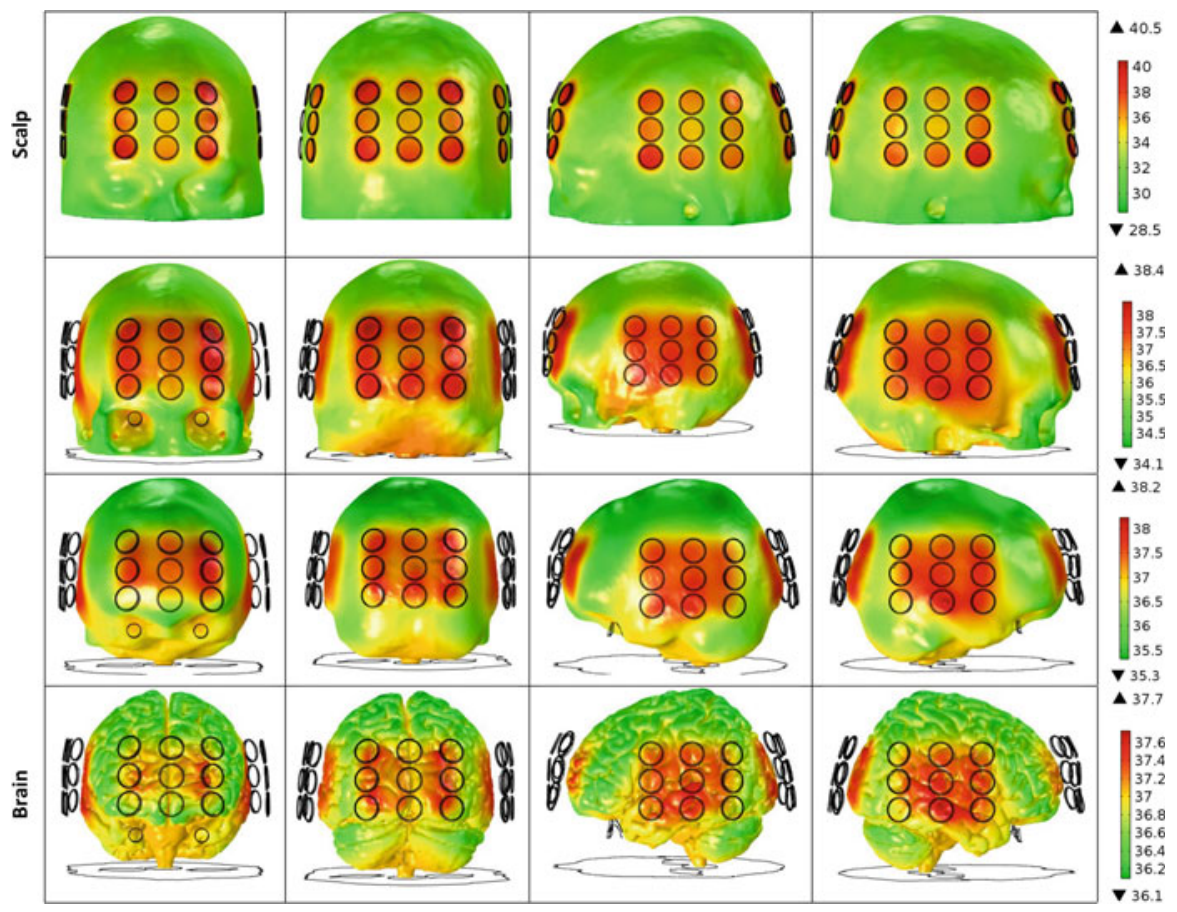

Fig. 9 Temperature distribution at the end of the simulation (360 s) on each tissue surface when current is decreased to $674 \mathrm{~mA}$ to avoid the need of shutting down the fields and considering sweat. First row, scalp; second row, skull; third row, CSF; fourth row, the brain. Note the different scales for each row. All values are in ${ }^{\circ} \mathrm{C}$

type of thermal studies in different head models and doing an experimental validation using phantoms would be useful to support our work.

Acknowledgements Instituto de Biofísica e Engenharia Biomédica is supported by Fundação para a Ciência e Tecnologia (FCT), Portugal, under grant n ${ }^{\circ}$ UID/BIO/00645/2013.

Conflict of Interests Pedro Cavaleiro Miranda has a research agreement funded by Novocure. Nichal Gentilal holds a $\mathrm{PhD}$ grant from the research agreement with Novocure.

\section{References}

1. Kirson, E. D., et al. (2004). Disruption of cancer cell replication by alternating electric fields. Cancer Research, 64, 3288-3295.

2. Kirson, E. D., et al. (2007). Alternating electric fields arrest cell proliferation in animal tumor models and human brain tumors. Proceedings of the National Academy of Sciences of the United States of America, 104, 10152-10157.

3. Tuszynski, J. A., et al. (2016). An overview of sub-cellular mechanisms involved in the action of TTFields. International Journal of Environmental Research and Public Health, 13, 1128. 
4. Carlson, K. W., et al. (2019). Simulating the effect of $200 \mathrm{kHz}$ AC electric fields on tumour cell structures to uncover the mechanism of a cancer therapy. In S. Makarov, M. Horner, \& G. Noetscher (Eds.), Brain and human body modeling. Cham: Springer.

5. Berkelmann, L., et al. (2019). Tumour-treating fields (TTFields): Investigations on the mechanism of action by electromagnetic exposure of cells in telophase/cytokinesis. Scientific Reports, 9, 7362.

6. Stupp, R., et al. (2012). NovoTTF-100A versus physician's choice chemotherapy in recurrent glioblastoma: A randomised phase III trial of a novel treatment modality. European Journal of Cancer, 48, 2192-2202.

7. Stupp, R., et al. (2015). Maintenance therapy with tumor-treating fields plus temozolomide vs temozolomide alone for glioblastoma: A randomized clinical trial. JAMA, 314, 2535-2543.

8. Stupp, R., et al. (2017). Effect of tumor-treating fields plus maintenance temozolomide vs maintenance temozolomide alone on survival in patients with glioblastoma: A randomized clinical trial. JAMA, 318, 2306-2316.

9. Ceresoli, G., et al. (2018). MA12.06 STELLAR - Final results of a phase 2 trial of TTFields with chemotherapy for first-line treatment of malignant pleural mesothelioma. Journal of Thoracic Oncology, 13, S397-S398.

10. Ballo, M. T., et al. (2019). Correlation of tumor treating fields dosimetry to survival outcomes in newly diagnosed glioblastoma: A large-scale numerical simulation-based analysis of data from the phase 3 EF-14 randomized trial. International Journal of Radiation Oncology, Biology, Physics, 104, 1106-1113.

11. Kanner, A. A., et al. (2014). Post Hoc analyses of intention-to-treat population in phase III comparison of NovoTTF-100A ${ }^{\mathrm{TM}}$ system versus best physician's choice chemotherapy. Seminars in Oncology, 5, S25-S34.

12. Toms, S. A., et al. (2019). Increased compliance with tumor treating fields therapy is prognostic for improved survival in the treatment of glioblastoma: A subgroup analysis of the EF-14 phase III trial. Journal of Neuro-Oncology, 141, 467-473.

13. Giladi, M., et al. (2015). Mitotic spindle disruption by alternating electric fields leads to improper chromosome segregation and mitotic catastrophe in cancer cells. Scientific Reports, $5,18046$.

14. Wenger, C., et al. (2016). Improving tumor treating fields treatment efficacy in patients with glioblastoma using personalized array layouts. International Journal of Radiation Oncology, Biology, Physics, 94, 1137-1143.

15. Korshoej, A., et al. (2018). Importance of electrode position for the distribution of tumor treating fields (TTFields) in a human brain. Identification of effective layouts through systematic analysis of array positions for multiple tumor locations. PLoS One, 13, e0201957.

16. Lacoutre, M. E., et al. (2014). Characterization and management of dermatologic adverse events with the NovoTTF-100A System, a novel anti-mitotic electric field device for the treatment of recurrent glioblastoma. Seminars in Oncology, 41, S1-S14.

17. Miranda, P. C., et al. (2013). The electric field in the cortex during transcranial current stimulation. NeuroImage, 70, 48-58.

18. Miranda, P. C., et al. (2014). Predicting the electric field distribution in the brain for the treatment of glioblastoma. Physics in Medicine and Biology, 59, 4137-4147.

19. Guyton, A., \& Hall, J. E. (2006). Text book of medical physiology (12th ed.). Philadelphia: Elsevier Saunders. Chapter 73.

20. Lim, C. L., Byrne, C., \& Lee, J. K. (2008). Human thermoregulation and measurement of body temperature in exercise and clinical settings. Annals of the Academy of Medicine, Singapore, 37, $347-353$.

21. Bergman, T. L., et al. (2011). Fundamentals of heat and mass transfer (7th ed.). New York: Wiley.

22. Hladky, S. B., \& Barrand, M. A. (2014). Mechanisms of fluid movement into, through and out of the brain: Evaluation of the evidence. Fluids Barriers CNS, 11, 26.

23. Wang, H., et al. (2014). Brain temperature and its fundamental properties: A review for clinical neuroscientists. Frontiers in Neuroscience, 8, 307. 
24. Pardridge, W. M. (2011). Drug transport in brain via the cerebrospinal fluid. Fluids Barriers CNS, $8,7$.

25. Shapiro, Y., Pandolf, K. B., \& Goldman, R. F. (1982). Predicting sweat loss response to exercise, environment and clothing. European Journal of Applied Physiology, 48, 83-96.

26. Givoni, B., \& Goldman, R. F. (1972). Predicting rectal temperature response to work, environment, and clothing. Journal of Applied Physiology, 32, 812-822.

27. ASHRAE. (2013). Fundamentals handbook. Atlanta: ASHRAE.

28. Gonzalez, R. R., et al. (2009). Expanded prediction equations of human sweat loss and water needs. Journal of Applied Physiology, 107, 379-388.

29. Harker, M. (2013). Psychological sweating: A systematic review focused on aetiology and cutaneous response. Skin Pharmacology and Physiology, 26, 92-100.

30. Pennes, H. (1948). Analysis of tissue and arterial blood temperatures in resting human forearm. Journal of Applied Physiology, 1, 93-133.

31. Gentilal, N., Salvador, R., \& Miranda, P. C. (2019). Temperature control in TTFields therapy of GBM: Impact on the duty cycle and tissue temperature. Physics in Medicine and Biology, 64, 225008.

32. Nathan, S. S., et al. (1993). Determination of current density distributions generated by electrical stimulation of the human cerebral cortex. Electroencephalography and Clinical Neurophysiology, 86, 183-192.

33. Sapareto, S. A., \& Dewey, W. C. (1984). Thermal dose determination in cancer therapy. International Journal of Radiation Oncology Biology Physics, 10, 787-800.

34. Dewhirst, M. W., et al. (2003). Basic principles of thermal dosimetry and thermal thresholds for tissue damage from hyperthermia. International Journal of Hyperthermia, 19, $267-294$.

35. Yarmolenko, P., et al. (2011). Thresholds for thermal damage to normal tissues: An update. International Journal of Hyperthermia, 27, 320-343.

36. Cserr, H. F. (1986). The neuronal microenvironment. Annals of the New York Academy of Sciences, 481, 123-134.

37. Moritz, A. R., \& Henriques, F. C. (1947). Studies of thermal injury: II. The relative importance of time and surface temperature in the causation of cutaneous burns. The American Journal of Pathology, 23, 695-720.

38. Lanier, W. L. (1995). Cerebral metabolic rate and hypothermia: Their relationship with ischemic neurologic injury. Journal of Neurosurgical Anesthesiology, 7, 216-221.

39. Rosomoff, H. L., \& Holaday, D. A. (1954). Cerebral blood flow and cerebral oxygen consumption during hypothermia. American Journal of Physiology, 179, 85-88.

40. Volgushev, M., et al. (2000). Synaptic transmission in the neocortex during reversible cooling. Neuroscience, 98, 9-22.

41. Volgushev, M., et al. (2004). Probability of transmitter release at neocortical synapses at different temperatures. Journal of Neurophysiology, 92, 212-220.

Open Access This chapter is licensed under the terms of the Creative Commons Attribution 4.0 International License (http://creativecommons.org/licenses/by/4.0/), which permits use, sharing, adaptation, distribution and reproduction in any medium or format, as long as you give appropriate credit to the original author(s) and the source, provide a link to the Creative Commons license and indicate if changes were made.

The images or other third party material in this chapter are included in the chapter's Creative Commons license, unless indicated otherwise in a credit line to the material. If material is not included in the chapter's Creative Commons license and your intended use is not permitted by statutory regulation or exceeds the permitted use, you will need to obtain permission directly from the copyright holder.

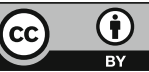

